\title{
DESIGN, FABRICATION, AND TEST OF AN SRF CRYOMODULE PROTOTYPE AT FERMILAB
}

\author{
W. Soyars, C. Darve, T. Nicol, A. Rowe \\ Fermi National Accelerator Laboratory \\ Batavia, IL, 60510, USA
}

\begin{abstract}
In support of the Charged Kaons at the Main Injector (CKM) experiment [1], an SRF cryomodule was designed, assembled, and tested at Fermilab. The cryomodule prototype consists of a single niobium 13-cell $3.9 \mathrm{GHz}$ superconducting RF cavity installed in its horizontal cryostat. The prototype was simplified to hold an additional dummy cavity in place of a second 13-cell SRF cavity. Although this cryomodule was originally intended for beamline deflection in the CKM experiment, this first preliminary test aims to compliment existing vertical 3-cell 3.9 GHz SRF cavity testing and also to gain expertise in the field of SRF testing. The cryomodule's thermal and mechanical design is reported. The test process and instrumentation is described. The first operational cooldown with RF powering is discussed and some cryogenic results are given.
\end{abstract}

\section{CRYOMODULE MECHANICAL DESCRIPTION}

A model of the RF cavity inside its superfluid helium containment vessel is shown in FIGURE 1. The assembly is mainly composed of a 13-cell RF cavity, a 6-inch titanium outer shell, and a tuner. This vessel holds 7.2 liters of superfluid He. The vessel features flanged connections that allow one to remove, process, and retest the same 13-cell cavity multiple times. A titanium bellows welded into the body of the cryostat allows the length to be stretched or compressed approximately $2 \mathrm{~mm}$ during cavity tuning at $1.8 \mathrm{~K}$. Initial tests were conducted without the tuner installed; a fixed bracket was substituted. Further mechanical design details are given elsewhere [2].

The maximum working internal pressure of the titanium cryostat, as limited by the bellows, is calculated to be $482 \mathrm{kPa}$ (70 psid). The test area relief system is configured to limit the internal differential pressure to $276 \mathrm{kPa}$ (40 psid) for the worst case failure of beam tube vacuum rupture, with air condensing on the bare inside surface of the niobium. The niobium RF cavity has been analyzed with $207 \mathrm{kPa}$ (30 psid) maximum external pressure, a point where detuning becomes a concern. 


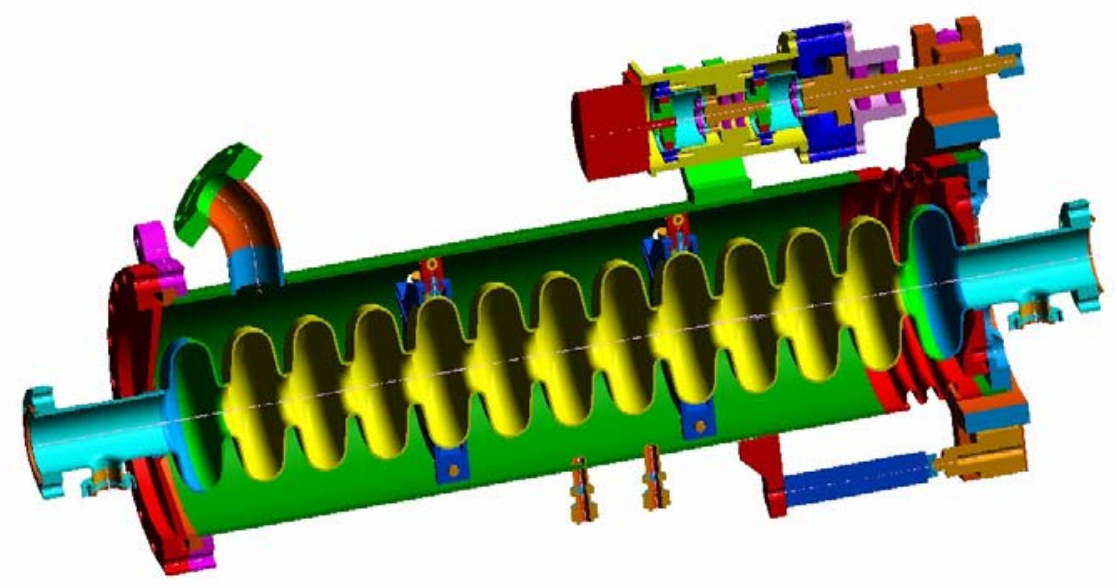

FIGURE 1. The CKM 13-cell cryostat cross-section with frequency tuner installed

The RF cavity as installed in the vacuum vessel is shown in FIGURE 2. While the original beam line design called for two 13-cell cavities within the cryomodule, this prototype only contains a single 13-cell cavity. A "place-holder" support structure takes the place of the second RF cavity. There are no current plans to install a second 13-cell cavity. The completed cryomodule at the test area is shown in FIGURE 3.

Magnetic shielding was mounted directly to the exterior of the superfluid helium vessel. This shielding is made out of Cryoperm (Amuneal Manufacturing Corp.), which possesses good magnetic shielding properties when at cryogenic temperatures. Room temperature measurements showed a mimimum terrestrial magnetic field attenuation of 20 . A LN2-cooled copper shield provides thermal shielding. The $2 \mathrm{~K}$ region was blanketed with 30 layers of multi-layer insulation while the $80 \mathrm{~K}$ region has 45 layers. There is no 5 K shield.

The stainless steel-to-titanium helium connections for the RF cavity vessel were sealed with DESY-style aluminum hex "diamond” seals. The cryogen supply connections to the test area use Parker Ultraseal fittings for the $1 / 4$ ” tubing connections.

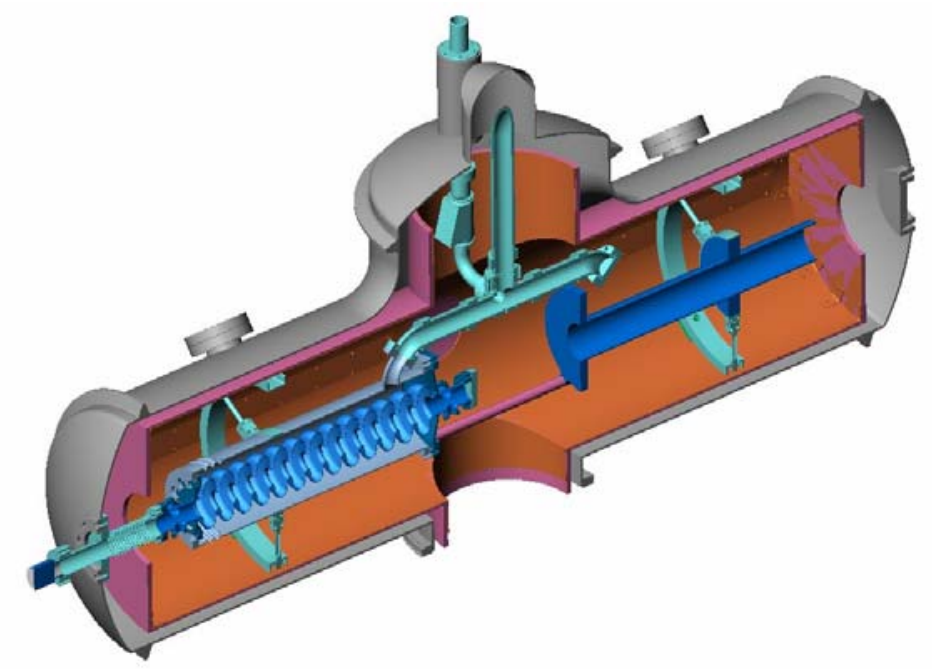

FIGURE 2. Prototype configuration of a single 13-cell RF cavity installed in vacuum vessel 


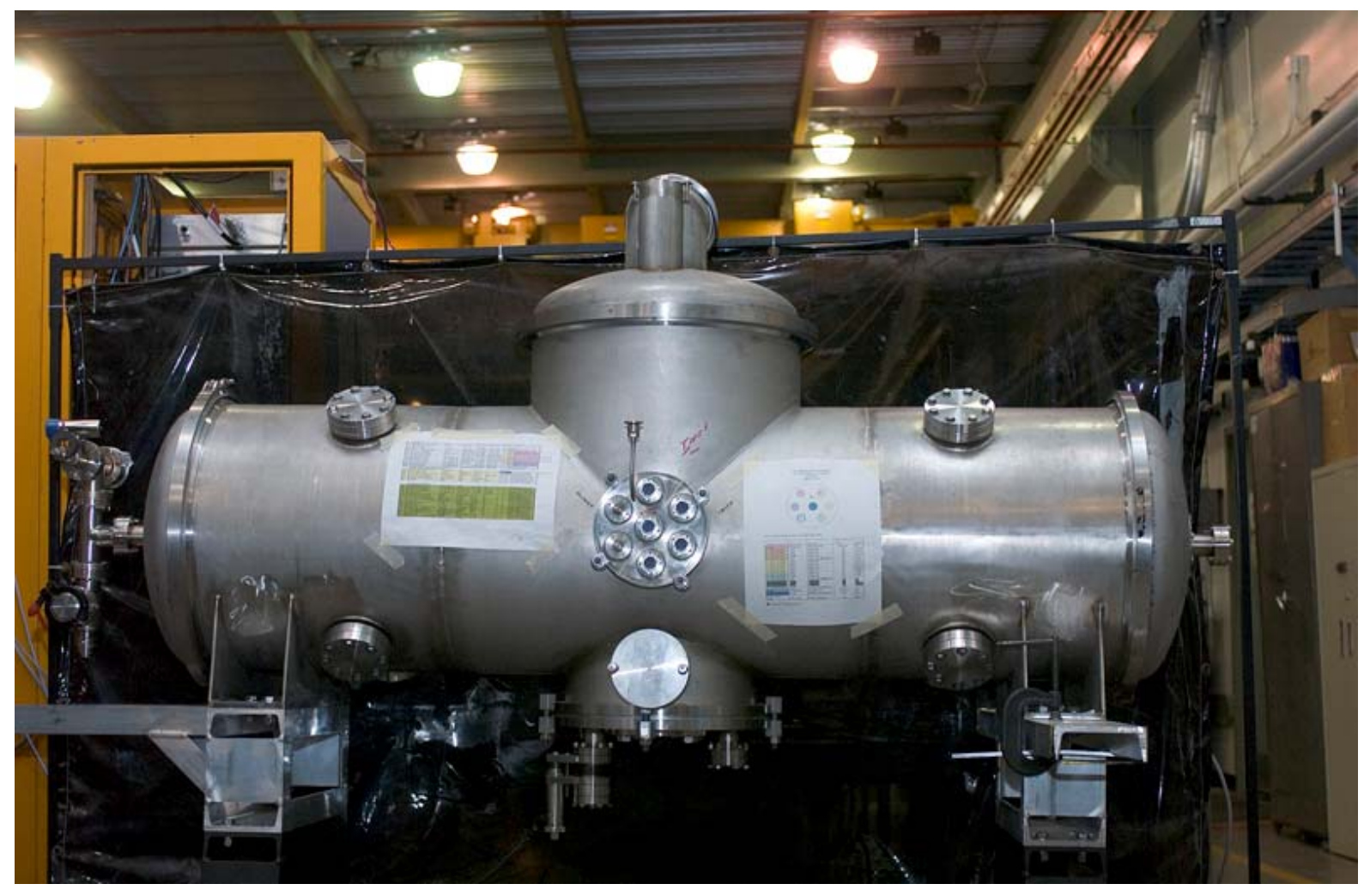

FIGURE 3. The CKM Cryomodule with 13-cell 3.9 GHz superconducting RF cavity

\section{CRYOGENIC SYSTEM FOR TESTING THE CRYOMODULE}

The A0 Superconducting RF Research and Development Test Area at Fermilab is a versatile, dewar-fed superfluid helium system for testing superconducting RF cavities [3]. Vacuum pumping capacity allows for about $2 \mathrm{~g} / \mathrm{s}$ of flow at 12 torr $(1.8 \mathrm{~K})$. This system usually is configured for testing bare RF cavities inside a Vertical Test Dewar. The CKM Cryomodule was installed here. The flow schematic for this setup is shown in FIGURE 4.

Temperature readings on the CKM Cryomodule are made by LakeShore Cernox and platinum sensors. Thermometers in the $4 \mathrm{~K}$ range use Lakeshore model CX-1050-SD, while the $2 \mathrm{~K}$ range uses model CX-1010-SD. The surface-reading sensors were mounted with varnish. Available control system open channels were very limited due to the fact that the A0 R\&D Test Area shares its control crate with other Fermilab facilities (Tevatron and FNPL Photoinjector) operations. Therefore, local display readings only were available for the cryomodule's Cernox resistors.

The liquid level control volume is provided with an American Magnetics (AMI) superconducting level probe. A $50 \mathrm{~W}$ cartridge heater is also mounted here for calorimetry purposes. Pressure measurements are made by Setra transducers of various ranges. Cryogenic flow is electrically controlled by a Fermilab-made valve (EVJV) and a Cryolab valve (EV19), both with an Industrial Devices actuator. The volumetric vacuum pump flow is electrically regulated with a Fermilab-made butterfly throttle valve (EVVC). 


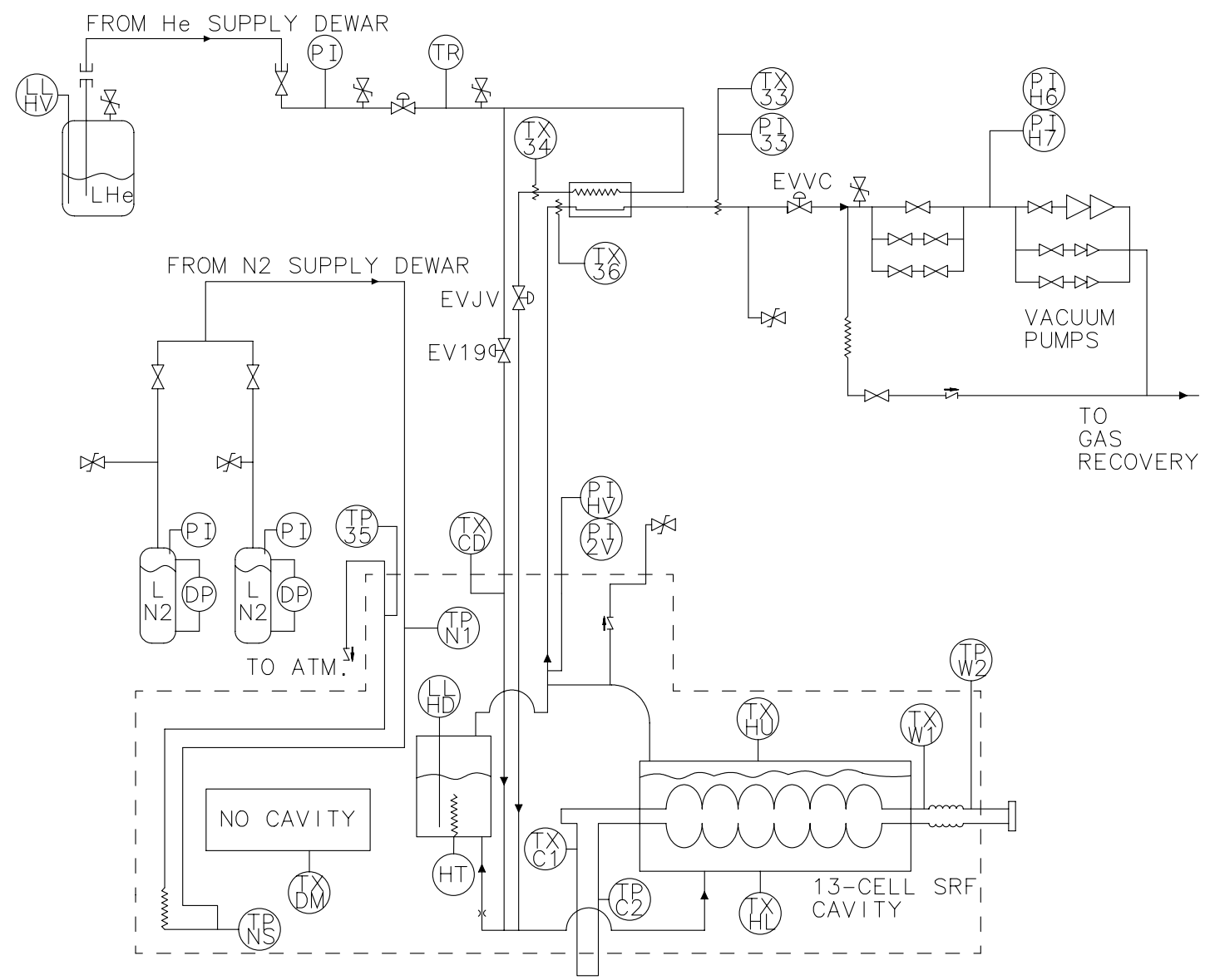

FIGURE 4. Flow schematic of CKM Cryomodule at A0 Superconducting RF R\&D Test Area.

\section{CRYOMODULE COOLDOWN}

Cooldown began on October 5, 2004. The supply transfer line and nitrogen thermal shield took 14 hours to reach $80 \mathrm{~K}$. At steady-state, 8 l/hr (1.8 g/s) of LN2 was supplied to the cryomodule. Since no control valve was available for throttling LN2 flow, the cryomodule $80 \mathrm{~K}$ heat load was not determined. One anomaly was that the $80 \mathrm{~K}$ coupler intercept point (TPC2) never got below 140K, indicating the need for a better thermal strap here. It is interesting that the coupler $80 \mathrm{~K}$ intercept point on the operational photoinjector SRF cavity ${ }^{3}$ also shows very similar behavior, indicating about $140 \mathrm{~K}$.

The helium vessel was initially cooled and held overnight to $20 \mathrm{~K}$, requiring $15 \mathrm{l} / \mathrm{hr}$ of dewar LHe usage. The cavity helium vessel was filled with $4.3 \mathrm{~K}$ LHe prior to testing in 30 minutes. At the conclusion of testing, the helium system was maintained in a $20 \mathrm{~K}$ "standby mode," requiring $9 \mathrm{l} / \mathrm{hr}$ inventory.

An anomaly discovered during cooldown was that the second "dummy" cavity (TXDM) never cooled off; it read $150 \mathrm{~K}$, and slightly decreasing, during testing. The original design intended this to be a liquid-containing second cavity "mock-up". When this was converted to being only a structural element since a second cavity is not planned, a thermal braid connection was mistakenly omitted. The four Invar tie rods (19 $\mathrm{mm} \mathrm{dia.} \mathrm{x}$ $376 \mathrm{~mm}$ long) connecting this structure to the active cavity did not provide adequate thermal connection to the cold mass. The abnormally warm dummy side contributed a conduction heat leak to the He vessel, estimated as about $2 \mathrm{~W}$ total. 


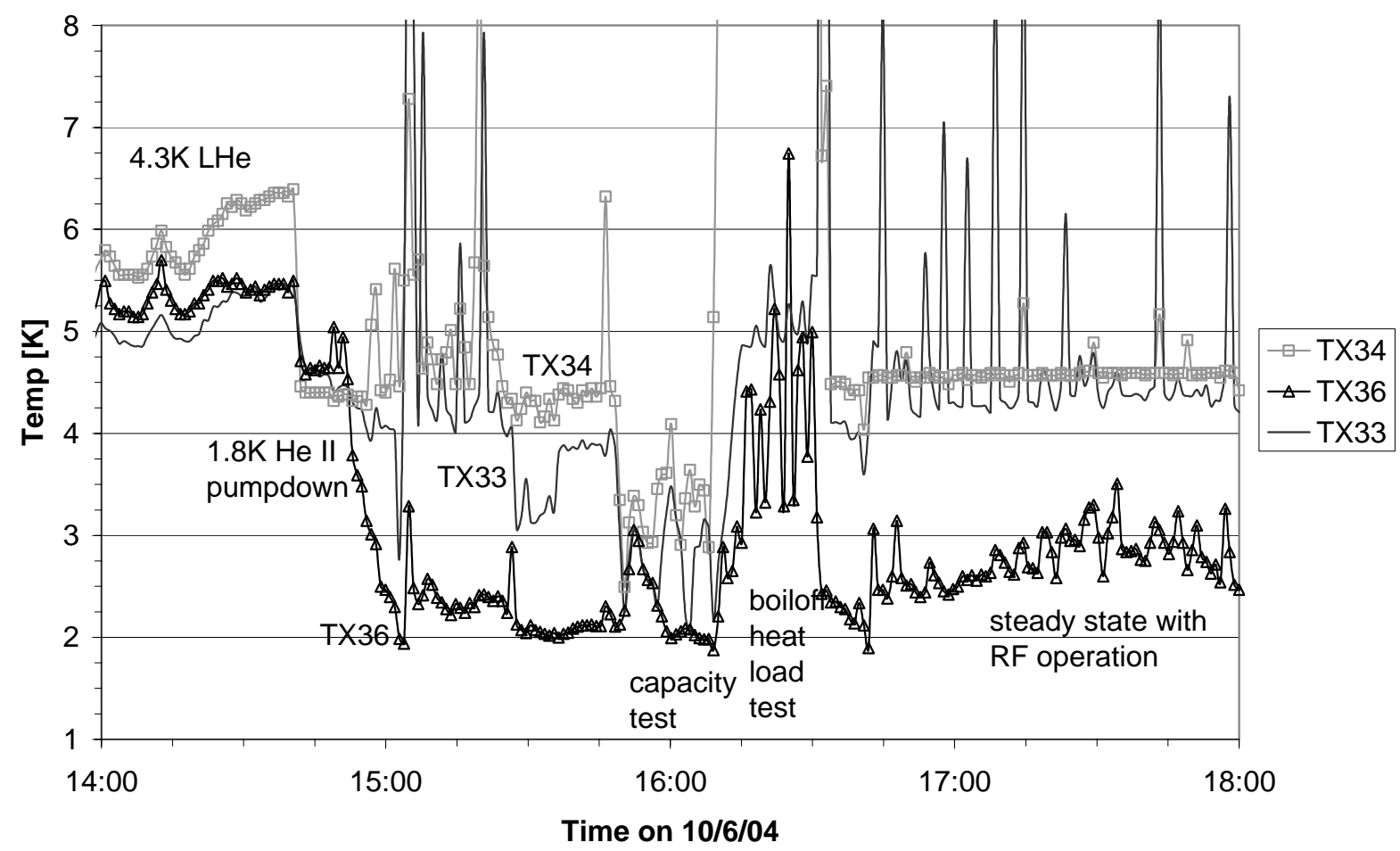

FIGURE 5. Helium fluid temperatures during superfluid cooldown and RF operation.

\section{CRYOMODULE 1.8 K SUPERFLUID OPERATION}

FIGURE 5 shows the period of superfluid filling and RF operation. LHe usage during the RF operation interval was $20 \mathrm{l} / \mathrm{hr}(0.63 \mathrm{~g} / \mathrm{s})$. Note that the high pressure exchanger outlet (TX34) is not subcooled; the heat exchanger duty of about $5 \mathrm{~W}$ is removing the helium transfer line heat leak.

\section{Transient (Boil-off) Heat Load Measurement}

During static heat load conditions, the supply flow was shut off, allowing the superfluid to boil off. Liquid volume as a function of liquid level was determined from CAD program solid model calculations for the helium vessel with the RF cavity inside. A series of heat load calculations were done using the change in vessel volume during the previous five minutes. These results as liquid level diminished are shown in FIGURE 6, indicating a static heat load to $1.8 \mathrm{~K}$ of $3.5+/-0.5 \mathrm{~W}$. As previously noted, this is higher than expected since the dummy side remained rather warm, creating about 1-2 W of extra heat load.

This same transient boil-off heat load method was used for a short period of "hard" RF operations (at 18:30) indicating a dynamic load of 10.0 +/- $1.0 \mathrm{~W}$ to $1.8 \mathrm{~K}$. Unfortunately, RF performance measurements were inconclusive for this period, so these are not available for comparison with cryogenic measurements. 


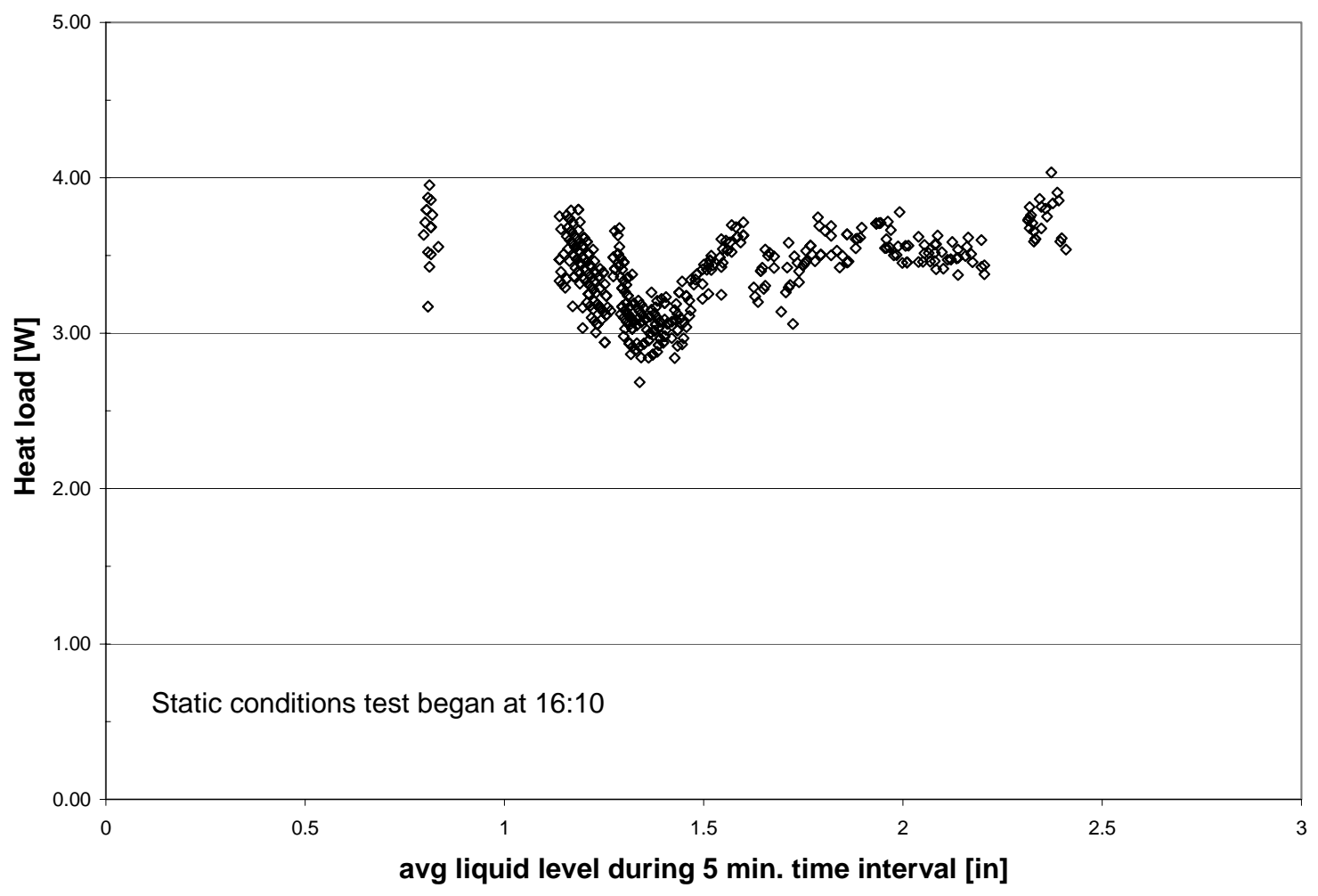

FIGURE 6. 1.8 K static heat load determination from transient boil-off.

\section{Steady-state Capacity Test}

Steady state tests were conducted with the superfluid supply valve (EVJV) maintaining a constant liquid level in the helium vessel. Vacuum pumping was maximized (no throttling with EVVC). Results are shown in FIGURE 7. With static heat leak only, the system achieved 6 torr minimum pressure. Applying the above static load measurements, this suggests total system capacity of $3.5 \mathrm{~W}$ at $1.62 \mathrm{~K}$ (6 torr).

Additional heat load to the superfluid helium bath was added by the electrical heater to simulate RF dynamic loading. With $5 \mathrm{~W}$ of heater power, we achieved steady state at 12 torr. This suggests $8.5 \mathrm{~W}$ total system capacity at $1.8 \mathrm{~K}$.

\section{CONCLUSIONS}

The design of an SRF cryomodule, the CKM Cryomodule, has been discussed. It was successfully cooled down to $1.8 \mathrm{~K}$ and its 13 -cell SRF cavity was powered. Experience was gained in the design, fabrication, and operation of an SRF cryomodule. The A0 Superconducting RF Research and Development Test Area system capacity was measured. The design issues and performance results will be helpful in guiding the development of future cryomodules and cryogenic systems. This cryomodule can be a test bed for future horizontal cavity and auxiliary system testing. 


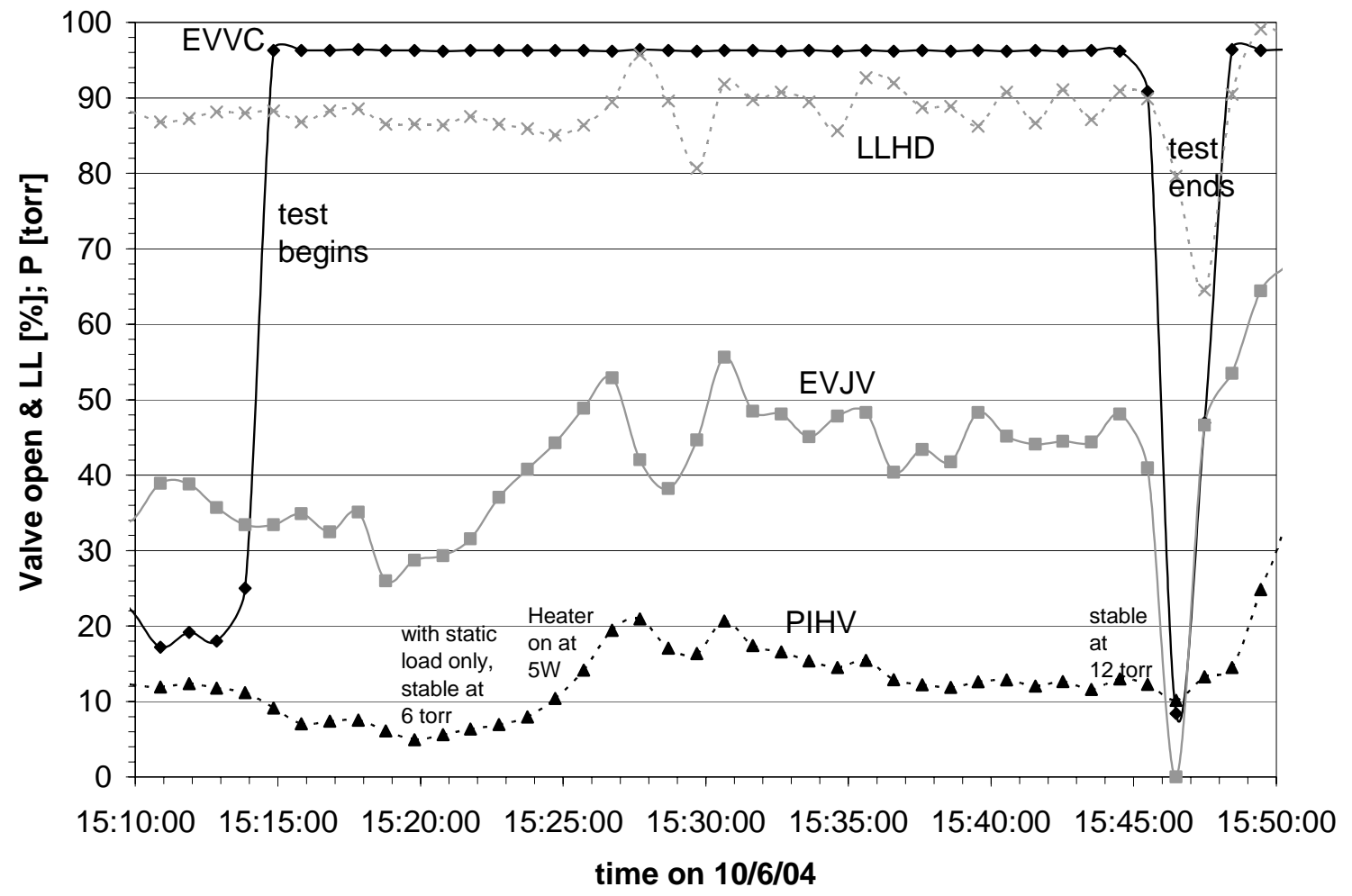

FIGURE 7. Capacity test with heater during steady-state conditions

\section{ACKNOWLEDGMENTS}

The authors wish to acknowledge Leo Bellantoni, the program physicist directing this work. The SRF vessel was designed by Peggy Crayton. Much of the initial engineering on the vacuum vessel and thermal shielding was done by Joel Fuerst. We received a great deal of help from Dave Erickson and his group during initial fabrication. The final fabrication and assembly team was Wilson Cross and Mike Barbor. The test installation was done by Wade Muryani and Mike Heinz. Brian DeGraff was very helpful during the test operations. The work was supported by the U. S. Department of Energy under contract No. DE AC02 76CH03000.

\section{REFERENCES}

1. Cooper, P. S., et. al. “CKM: Charged Kaons at the Main Injector,” Nucl. Phys. Proc. Suppl. 99B:121126 (2001).

2. Rowe, A. "Prototype CKM Titanium Cryostat \& Niobium SCRF 3.9 GHz Cavity Fabrication Description and MAWP Justification”, Fermilab memo, September 2, 2004.

3. DeGraff, B. D., Soyars, W. M., and Martinez, A. "SRF Cryogenic Operating Experience at FNPL,” to be presented at 2005 CEC. 